\title{
Neuer Beitrag zur Kenntnis der Vogelwelt der Provinz Posen.
}

\author{
Von Professor J. Hammling.
}

(Soblufs von S. 1-42.)

178. Certhia familiaris L. - Waldbaumläufer.

In meinen früheren Berichten (I. und II.) hatte ich die beiden bei uns vorkommenden Formen dieser Art nicht gesondert behandelt, wohl aber dem Gesange nach unterschieden. Ich vermutete in dem Urheber der kürzeren Strophe (titiroiti) c. familiaris, während ich in dem Urheber der lauteren Strophe brachydactyla erkennen zu müssen glaubte und zwar deswegen, weil die erste Form, die oft als die westliche bezeichnet wird, hier viel häufiger angetroffen wird als die zweite, die östliche, während man doch eher das Gegenteil hätte erwarten sollen. Meine Vermutung war irrtümlich, und Professor Voigt machte mich in dankenswerter Weise auf diesen Irrtum aufmerksam. Auch Dobbricks Schrift „Unsere Baumläuferarten“ (Jahrb. des westpreufs. Lehrervereins für Naturkunde, 1913) wirkte aufklärend. Man vergleiche auch den neuerdings erschienenen Artikel Fenks „Zum Gesang von Certhia" (0. Mb. 1915, No. 11, S. 170 ff.). Das beste Unterscheidungsmerkmal beider Certhia-Formen ist der Gesang. Alle übrigen (z. B. Färbung und sonstige Stimmäufserungen) sind für den Feldornithologen von geringerer Bedeutung, weil die Vögel sich wegen ihrer Unruhe meist einer eingehenden Betrachtung entziehen und ihre Rufe doch wohl manches Übereinstimmende haben. Mir wenigstens ist bisher eine sichere Unterscheidung der Rufe beider Formen nicht gelungen. Die Gesänge zeigen allerdings einen meist recht verschiedenen Charakter. Das Liedchen von c. familiaris ist in "Klangfarbe und Aufbau" (Fenk) anders als das von brachydactyla. Jenes wird lauter, d. h. mit gröfserer Stimmkraft vorgetragen und ist meist durch einen dem des Blaumeischens ähnlichen Triller ausgezeichnet. Ich werde nunmehr die Fälle, in denen ich diese Form (c. familiaris) bei Posen beobachtet habe, aufführen und dabei einige Liedchen, wie ich sie gehört habe, in Buchstaben wiedergeben. Es sind nicht allzu viele Fälle, denn, wie ich schon oben sagte, diese Form ist hier entschieden seltener als die andere.

Am 22. IV. sang $1 \sigma^{\top}$ in den Kernwerksanlagen zitiroitiroizirrr. Der Triller liegt hier am Ende des Liedchens, das im übrigen eine gewisse Åhnlichkeit mit der Strophe der andern Form erkennen lärst. Am 18. III. 1906 hörte ich auf dem Petrikirchhofe die Strophe zizitirrroazitiroi, mit dem Triller in der Mitte, was in der Regel der Fall ist. Am 15. IV. 1907 sang $1 \sigma^{t}$ in den Kernwerksanlagen zizizi (abfallend) zizizirrrlelaziowit; in diesem 
Falle handelte es sich um ein Brutpärchen, das auf der Wohnungssuche war; am 17. IV. hörte ich eine ähnliche Strophe im Eichwalde; ebendort auch am 10. V., vielleicht von demselben $\sigma^{x}$. Am 18. II. und am 18. III. $19081 \sigma^{x}$ auf dem Petrikirchbofe singend; am 20. III. am Südrande des Eichwaldes 1 Stück. Am 22. V. 1909 im Eichwalde und ebendort am 27. V.; auch in diesem Falle handelte es sich wohl um $1 \sigma^{x}$. Am 7. IV. 1910 an der Wartheseite der Kernwerksanlagen 1 singendes Stück; am 17. V. in Lubasch (Kr. Czarnikau) $1 \sigma^{x}$. Am 24. V. $1 \sigma^{x}$ eifrig singend im Eichwalde unter den alten Eichen südlich des Bahndammes; ebendort am 27. V. dasselbe $\sigma^{x}$; am 26. V. $1 \sigma^{x}$ singend an der Nordseite des Kernwerks. Am 19. III. $19121 \sigma^{7}$ an der nördlichen Randlache des Eichwaldes; dasselbe $\sigma^{\top}$ sang auch am 26. III. und am 26. IV.; am 24. IV. 1 \% auf den Schiefsständen vor dem Warschauer Tor (zizizilahoit, obne Triller !). Am 14. IV. $19131 \sigma^{x}$ in der Nähe des Lubascher Sees; 19. V. im Eichwalde 1 Stück; 1. VI. $1 \sigma^{x}$ singend unter den hohen Kiefern unfern des Dembitsch-Sees bei Krummfliefs (Kr. Posen Ost), meist mit einem Triller beginnend. Am 18. III. 1914 1 Stück an der Westseite des Eichwaldes, ebendort am 16. V.; am 6. VI. wieder 1 Stück an andrer Stelle im Eichwalde singend. Diese Form wurde also bei Posen in den Kernwerksanlagen, auf dem Petrikirchhofe, im Eichwalde, auf den Schielsständen vor dem Warschauer Tor, ferner bei Lubasch und in der Forst Krummfliefs beobachtet, doch immer nur einzeln, so dafs also ihr Auftreten bei Posen als ziemlich selten bezeichnet werden mufs. Die Aufenthaltsorte trugen waldartigen Charakter; oft, nicht immer, waren Kiefern unter Laubbäumen eingestreut oder es wucherte unter Kiefern dichtes Unterholz. Dals ein fam. ơ je die Strophe von brach. wiedergegeben hätte, ist von mir hier nicht beobachtet worden.

\section{Certhia brachydactyla Brehm. - Kurzzehiger oder Gartenbaumläufer.}

Diese Form des Baumläufers kommt bei Posen neben der andern vor, und zwar ist sie die häufigere. Weil beide Formen in denselben Gegenden neben einander leben, erkennen ihnen manche Ornithologen den Wert guter Arten zu (Kollibay a. a. 0., S. 294 ; R. Fenk in den 0. Mb. 1915, S. 170).

Die typische Gesangsstrophe dieser Form ist meist kürzer und wird mit einer dünneren, feineren Stimme vorgetragen. Sie lautet tiroiti oder ziroiti; die vorletzte oder auch die letzte Silbe ist kräftiger betont. Dieses Liedchen wird nicht selten erweitert, indem die erste Silbe 2 oder $3 \mathrm{mal}$ angeschlagen wird: titiroiti oder tititiroiti. Manche $\sigma^{\top}$ lassen auch wohl noch ein 4 ti hören oder verdoppeln die Strophe: titititiroiti (am 11. II. 1910 auf dem Grünen Platze, jetzt Ludendorffplatz) oder tiroititiroiti (am 2. IX. 
1910 im Kernwerk); am 10. XI. 1905 wiederholte $1 \sigma^{\prime \prime}$ im Kernwerk seine Strophe sogar dreimal: titiroitititiroitititiroiti. So wurde zwar dus Liedchen ziemlich lang, aber diese Verlängerung bestand nur in der mehrmaligen Wiederholung der typischen Strophe, ohne dafs der Aufbau der Strophe eine Änderung erfahren hätte. Der Gesang dieser Form wurde nicht selten schon im Februar vernommen.

Der kurzzehige Baumläufer wurde in der Umgebung Posens an folgenden Stellen beobachtet: In der Nähe von Golencin; im Eichwalde; im Kernwerk meist mehrere Pärchen; im Schilling; an der Eichwaldstrafse; im Kobylepoler Grunde; im Garten des Krankenhauses am Bernhardinerplatz; im Viktoriapark; auf den Pappeln des Weges von Golencin nach der Elsenmühle (Familie, das $\sigma^{x}$ sang); auf dem Petrikirchhofe; am Schlofspark Ostend; auf dem Turnplatze und im botanischen Garten des MarienGymnasiums; auf den Pappeln am Schillingstor; auf dem Kirchhofe vor dem Warschauer Tor; im Park von Kobylepole; auf dem Kirchhofe an der Garnisonkirche; in der Nähe des GneisenauDenkmals. Hiernach verdient diese Form in der Tat den Namen Gartenbaumläufer.

Bruten wurden mehrfach beobachtet; alte Vögel, die mit ihren Jungen umherzogen, wobei die alten of oft ihr Liedchen hören liefsen, wurden nicht selten gesehen.

Am 18. VI. 1914 hörte ich $1 \sigma^{\pi}$ in einigen Kiefern am rechten Wartheufer gegenüber dem Warthewalde unweit des Fährhauses von Czeszewo (Kr. Wreschen).

Nach Kayser sind beide Formen bei Lissa ziemlich spärlich vorhanden.

\section{Sitta caesia Wolf. - Kleiber.}

181. Sitta caesia sordida Rchw. - Ostdeutscher Kleiber.

Bei Posen scheinen beide Formen des Kleibers vorzukommen, soweit sich dies durch eine Beobachtung der Vögel in der freien Natur überhaupt entscheiden läfst. Jedesfalls sind Stücke mit ockergelber (besonders im Herbst), sowie mit mehr oder weniger weifser, ockergelblich verwaschener Unterseite oft beobachtet worden. Die im Bericht II. im Schilling beobachteten Brutvögel, sowie andere im Eichwalde gesehene Stücke gehörten anscheinend zu sordida. Die Verbreitung der Formen des Kleibers in der Provinz ist zurzeit noch durchaus unsicher.

\section{Parus maior L. - Kohlmeise.}

Am 29. V. 1914 hatte ein Pärchen in einem an der Aufsenwand des Eichwaldrestaurants angebrachten tönernen Rehkopfe 12 Junge. Zwei Jahre vorher war hier die Brut dadurch gestört worden, dals das $\subsetneq$ durch Unachtsamkeit getötet worden war. 


\section{Parus ater L. - Tannenmeise.}

Während ich früher (vgl. I.) die Tannenmeise nur in der Strichzeit im Eichwalde antraf, konnte ich den Vogel am 20. IV. 1912 auch in der Brutzeit daselbst feststellen und $\mathrm{zwar}$ in den Kiefern südlich des Försterhauses. Am 13. VI. machte sich 1 Stück in den hohen Kiefern am Südrande des Eichwaldes durch Rufe bemerkbar.

\section{Parus palustris L. - Glanzköpfige Sumpfmeise.}

Am 1. III. 1914 rief im Goethepark 1 Stück mehrmals tschewíhp tschewíbp, dann etwas später tjihp - - - meist viermal, doch auch manchmal nur zweimal; dann ging das Meischen über zu den Rufen tjip _- _ _ - wiederholte aber dazwischen auch hin und wieder die zweite Rufform.

Die mattköpfige Form habe ich hier bisher nicht beobachtet, auch Kayser bei Lissa nicht.

\section{Parus cristatus L. - Haubenmeise.}

1913: Am 21. IV. an 2 Stellen in den Kiefern oberhalb des Kobylepoler Grundes rufend; am 25. IV. war an 2 Stellen des Kiefernwaldes jenseit Kobylepole das Zi zi zi zürrr und Zi gürrr von Haubenmeisen zu hören.

1914: Am 14. II. 1 Stück rufend im Solatscher Wäldchen. Am 9. VII. beobachtete ich diese Art bei Antonin und vorher am 7. VII. bei Przygodzice.

Es dürfte sich um die Form mitratus gehandelt haben; Belegexemplare habe ich jedoch bisher nicht in Händen gehabt.

Nach Kayser zuweilen bei Lissa beobachtet.

\section{Aegithalus caudatus L. - Schwanzmeise.}

Diese Art ist nur spärlicher Brutvogel bei Posen (vgl. I.).

1912: Am 7. I. I Stück um 4 Uhr p. an der Wartheseite des Kernwerks lebhaft zit $-\ldots-$ rufend; am 17. V. ebendort. Am 22. VI. eine Familie im Eichwalde; ebendort am 20. XII. eine Familie, 6 Stücke sichtbar, zerr rufend, dazwischen ein leises Tik. vertreten.

Auch bei Lissa ist diese Art nach Kayser ziemlich spärlich

\section{Accentor modularis L. - Heckenbraunelle.}

Nach A. v. Homeyer war die Heckenbraunelle in Zerkow und an der Prosna im Oktober und November 1863 häufig.

Diese Art überwintert manchmal bei uns, so bei Bromberg und auch bei Posen. Bei Bromberg wurde die Braunelle von K. Kothe und Schiller im Winter angetroffen (J. f. O. 1910, 4. Heft, S. 814); in Posen am 22. II. 1903 1 Stück im Weidengebüsch der 
Warthe unweit des Schillings (I.). Als Brutvogel ist die Heckenbraunelle, wie es scheint, im Posenschen noch nicht festgestellt worden.

\section{Sylvia nisoria Bchst. - Sperbergrasmücke.}

Diese Art wurde von Schwaitzer bei Wittowo (Kr. Schroda), von A. v. Homeyer an der Prosna beobachtet.

1912: Auch in diesem Jahre bei Posen häufig. Den ersten Vogel hörte ich am 11. V. um 4 Uhr p. in den Festungsanlagen am „Städchen“; am 13. V. 1 Stück am Eingange zum Schilling. Etwas häufiger waren die Vögel am 14., zahlreich vorhanden vom 15. ab an allen zusagenden Plätzen. Der Gesang wurde bis tief in den Juni hinein vernommen, doch nahm die Sangeslust von der Mitte dieses Monats an merklich ab. Letzter Gesang am 26. VI.

1913: Am 9. V. um $7^{10} \mathrm{Uhr}$ p. singend in einem dichten Weidenbusche diesseit der Loncz-Mühle; im Cybinatal jenseit Kobylepole noch fehlend; am 12. V. $1 \sigma^{x}$ an der Westecke des „Rohrteichs". Erst vom 14. V. an zahlreicher vorhanden und zwar an der Südseite des Eichwaldes $2 \sigma^{x}$; an der Warthe in der Höhe des Viktoriaparks $1 \sigma^{x}$; in Johannistal $2 \sigma^{x}$; diesseit der Loncz-Mühle $2 \sigma^{x}$; an der Südseite des Kernwerks am „Rohrteich" $4 \sigma^{7}$; in den Anlagen an der Oborniker Chaussee $1 \sigma^{\text {t }}$ (einen schönen Balzflug ausführend); an der militärischen Strafse in der Nähe der Kläranlage $2 \sigma^{\Upsilon}$; an der Cybinamündung $1 \sigma^{\star}$; an der Warthe in der Höhe der Wolfsmühle 2-3 $\sigma^{\nwarrow}$; im Schillerpark oder auf dem angrenzenden Kirchhofe $10^{7}$, das am 30. V. die einleitenden Töne aus dem Gesange des Gartenrötlings nachahmte; in den Festungsanlagen am "Städtchen" mehrere ơ.

Sonstiges Vorkommen: Am 14. V. traf ich im Obrabruche, besouders zwischen dem Pruth - Kanal und dem Obra-Nordkanal neben zahlreichen Dorngrasmücken auch mehrere Sperbergrasmücken, die auf den Wegbäumen eifrig sangen. In den feuchten Strafsengräben standen niedrige, von Lupulus durchzogene Weidenbüsche, und der die Strafse begleitende Bahndamm war mit dichtem Weidengebüsch bestanden. Hier dürften die Vögel gebrütet haben. Ein weiteres $\sigma^{7}$ beobachtete ich im Teufelszwirn gegenüber dem Bahnhofsgebäude in Kosten.

1914: Am 17. V. mehrere Stücke in den Festungsanlagen am „Städtchen" singend; am 19. V. $1 \sigma^{x}$ eifrig singend in dem buschreichen Garten diesseit des Solatscher Gutsparks und in den neuen Solatscher Anlagen (Balzflug). Am 30. V. hörte und sah ich ein Pärchen in einem Weidenbusche am Schwersenzer See. Am 6. VI. sangen an der Südseite des ehemaligen Rennplatzes 3 ơ.

Am 3. VII. traf ich 1 Stück in der "Bagna" im Kreise Obornik, das sein warnendes Terr hören liefs.

1915: Am 17. V. mehrere Stücke an der Wartheseite des Viktoriaparks singend. 
Am 25. V. traf ich am Moschiner Obra-Kanal 8 bis 10 Stücke, die eifrig sangen. Auch Balzflüge wurden von einzelnen Stücken wahrgenommen. Dagegen waren die vor 2 Jahren am Bahndamm nistenden Vögel verschwunden, weil die Weidenbüsche am Bahnkörper niedergelegt waren (vgl. O. Mb. 1916, Maiheft).

Nach Kayser ist diese Art bei Lissa selten.

Ein Stück aus Altkloster (Kr. Bomst) befindet sich nach Dr. Hesse (a. a. O., S. 601) im Berl. Mus.: No. B 481. 24. V. 1899. Altkloster (Posen) (Geschlechtsbestimmung fehlt).

Sperbergrasmücken wurden, abgesehen von der Umgebung Posens, demnach an folgenden Stellen beobachtet: In der Nähe von Guhren im Kreise Czarnikau; bei Wittowo (Kr. Schroda); an der Prosna; im Obrabruche bei Kosten; in der Bagna (Hochmoor) im Kreise Obornik; in der Nähe der Stadt Kosten; in der Nähe von Lissa; bei Altkloster im Kreise Bomst.

\section{Sylvia borin Bodd. - Gartengrasmücke.}

1912: Erst am 15. V. hörte ich um $6^{20}$ Uhr p. 1 Stück im Kobylepoler Grunde; am 17. daselbst 2 singende $\sigma^{\Upsilon}$.

1913: Am 6. V. Gesang von dieser Art in einer kleinen Lichtung in den hohen Kiefern der Südseite des Eichwaldes. Als ich mich dem Vogel näherte, dämpfte er sein Liedchen zu einem lieblichen Flüstern. Am 7. V. sang 1 Stück trotz des kalten Nordostwindes an der Wartheseite des Kernwerks.

In der Provinz beobachtet (vgl. auch 1I.): Am 14. V. in einem kleinen Erlenhain am Obrabruch bei Bonikowo (Kr. Kosten) mindestens 3 singende $\sigma^{x}$; am 17. V. am See von Kowalskie im Tale der Gluwna; am 1. VI. am Dembitsch-See in der Promnoer Forst unweit Krummfliefs mehrere singende Stücke; am 24. VI. mehrere $\sigma^{7}$ bei Lubasch (Kr. Czarnikau).

1914: Am 7. V. $1 \delta^{x}$ an der Rückseite des Viktoriaparks singend, ein 2 tes in der Lichtung an der Nordseite des Eichwaldes; am 20. V. 2 Stücke singend am Steilufer der Warthe.

Am 2. VI. hörte ich diese Art auf der Maiglöckcheninsel im Primenter See (Kr. Bomst); am 18. VI. reichlich im Warthewalde; am 6. VII. singend bei Antonin, am 7. VII. in der Nähe des Bahnhofs Przygodzice und unweit der Waldwärterei Tarchalski (mehrere $0^{\top}$ ).

1915: Am 8. V. singend im Eichwalde. Am 15. VII. daselbst noch Gesang an 2 Stellen.

Nach Kayser bei Lissa ziemlich spärlich vertreten.

190. Sylvia communis Lath. - Dorngrasmücke.

1912: Ankunft infolge der kühlen Nordost- und Ostwinde verzögert; am 5. V. hörte ich das erste Stück um 7 Uhr p. vor dem Warschauer Tor singen; am 6. V. 1 Stück an der mittleren Unterführung im Eichwalde; am 7. V. ziemlich zahlreich, häufig erst vom 14. V. an. 
Am 4. VI. eifriger Gesang eines $\sigma^{7}$ in einem Roggenstücke unweit der Pionierkaserne; hier und in einem benachbarten Weizenfelde sang das $\sigma^{T}$ auch am 11., 22., 24. V1. und am 2. VII.

1913: Am 30. IV. ein singendes $\sigma^{\top}$ am rechten Wartheufer oberhalb des Schillings, ein zweites an der Warthe in der Höhe der Gärtnerei von Zippel, ein drittes am Steilufer der Warthe.

Am 3. VI. sang 1 Stück in dem kleinen, aber buschreichen Garten der Garnisonwaschanstalt. Am 5. VI. wieder der Gesang eines Stückes in einem Roggenstücke am Wiesenwege nach dem Eichwalde.

In der Provinz beobachtet: 14. V. im Obrabruche bei Bonikowo reichlich; 17. V. diesseit Wierzonka auf dürrem Gelände, das aber einige Schwarzdornbüsche trug, häufiger im nahen Gluwnatale; 1. VI. unweit des Dembitsch-Sees bei Krummfliefs in dem dichten Unterholz unter hohen Kiefern; 24. VI. mehrfach bei Lubasch; 4. VII. singend in der Umgebung von Powidz (Kr. Witkowo); im Netztal bei Guhren nicht selten.

1914: Am 30. IV. 1 singendes $\sigma^{\top}$ an der rechten Wartheseite gegenüber dem Schilling; am 6. V. schon ziemlich reichlich an der Wartheinsel.

Am 2. VI. Gesang dieser Art auf der Maiglöckcheninsel im Primenter See; am 3. VII. singend in der Bagna im Kreise Obornik.

Am 1. VII. sang 1 Stück an der Bogdanka diesseit der Solatscher Anlagen in einem Haferfelde.

Nach Kayser bei Lissa nicht selten, berührt jedoch nicht das eigentliche Stadtgebiet.

\section{Sylvia curruca L. - Zaungrasmücke.}

1912: Am 22. IV $1 \sigma^{*}$ eifrig singend gegen $1 / 86$ p. an der Wartheseite des Kernwerks; am 24. IV. waren schon mehrere zu hören, aber noch immer keine andere Grasmücke; erst am 1. V. machte dem Müllerchen ein Plattmönch im Kernwerk Konkurrenz.

1913: Am 24. IV. sang 1 Stück gegen 4 Uhr p. am Steilufer der Warthe; am 25. IV. an 2 Stellen an der Südseite des Kernwerks singend.

Am 9. V. fand ich ein Nest in einer lichten Kiefernschonung jenseit Kobylepole. Es stand auf einigen trockenen Reisern zwischen ein paar Kiefernstämmchen etwa $1 / \mathrm{m}$ über dem Erdboden. Das $\sigma^{\pi}$ zeigte sich sehr beunruhigt und liefs bei meiner Annäherung mehrmals seinen Gesang hören. Das Nest, das noch keine Eier enthielt, wurde infolge der Störung verlassen.

Sonstiges Vorkommen: Am 17. V. im Gluwnatale bei Barcinek in einer Kiefernschonung singend; ebenso bei Kobylepole und bei Unterberg mehrfach in Kiefern; 1. VI. unweit des Dembitsch-Sees bei Krummfliefs; 24. VI. bei Lubasch. 
1914: Am 19. IV. liefs 1 o mehrmals im Park von Kobylepole den lauten Teil seines Gesanges hören; am 20. IV. singend am Eingange zum Schilling; am 22. IV. mehrfach gehört, während von andern Grasmücken noch keine wahrgenommen wurde.

Am 18. VI. hörte ich 1 Stück in der Nähe von Zerkow (Kr. Jarotschin). Nach Kayser nicht selten bei Lissa, aber weniger häufig als die folgende Art.

\section{Sylvia atricapilla L. - Mönchgrasmücke.}

1912: Am 1. V. 1 s singend an der Wartheseite des Kernwerks unweit des Schillingstors; erst am 7. V. etwas häufiger.

Am 18. IX. war im Schilling 1 Stück deutlich sichtbar, während ein zweites sich durch Rufe bemerkbar machte.

1913: Am 26. IV. $1 \sigma^{x}$ singend an der Südseite des Kernwerks gegenüber dem Bahnhof Gerberdamm; am 27. IV. singend im Kobylepoler Grunde.

Am 28. V. ahmte $1 \sigma^{T}$ am Solatscher Teiche deutlich mehrere Motive aus dem Gesange des Gartensängers nach und liefs, allerdings leise, den Schreckruf der Amsel hören.

Am 12. VIII. machte ein junges $\sigma^{r}$ im Schillerpark Gesangsübungen; am 18. VIII. sang um $1 / 2,1$ Uhr mittags ein junges Stück im Vorgarten des Mariengymnasiums; derselbe Vogel sang am 22. VIII. um $1 /, 9$ a. auf dem Schulhofe und später wieder im Vorgarten.

Sonstiges Vorkommen: Am 17. V. im Gluwnatale am See von Kowalskie; am 1. VI. am Dembitsch-See bei Krummfliefs; am 24. VI. bei Lubasch.

1914: Am 26. IV. brachte an der Wartheseite des Kernwerks $1 \sigma^{\pi}$ nach mehreren mifsglückten Versuchen den Überschlag ziemlich gut heraus.

Am 18. V. liefs $1 \sigma^{x}$ an der Südseite des Kernwerks 3 oder 4 mal ein drosselartiges Tüdí - - - hören und fuhr dann jedesmal in seinem eigenen Gesange (Überschlag) fort. Am 31. V. begann $1 \sigma^{t}$ auf dem Petrikirchhofe mehrmals seineu Überschlag mit 2 bis 4 oder 5 Tönen der Amsel, also manchmal mit einer ganzen Strophe derselben und brachte dann den Gesang in seiner Art zu Ende. In diesen Fällen wurde das einleitende Grasmückengeplauder nicht gehört, was ja freilich auch sonst bei vorgeschrittener Sangeszeit oft, ja fast immer fortgelassen wird. Am 3. VI. begann auch im Eichwalde $1 \sigma^{x}$ seinen Gesang mit einer Amselstrophe.

Am 2. VI. traf ich diese Art auf der Maiglöckcheninsel im Primenter See, bei Neudorf - Mauche und bei Oelpoche (Kr. Bdomst); am 18. VI. sangen zahlreiche Stücke im Warthewalde; am 6. und 8. VII. an mehreren Stellen im Park von Antonin und am 7. VII. am Bahnhof Przygodzice und im nahen Mischwalde singend. 
Never Beitrag zur Kenntnis der Vogelwelt der Provinz Posen. 199 gewandt.

1915: Am 30. IV. singend im Kernwerk, noch etwas un-

Noch am 15. VII. und wieder am 16. und 17. VII. sang 1 Stück auf dem Petrikirchhofe.

Der Abzug im Herbste geht manchmal recht spät vor sich. Noch am 3. X. 1915 traf ich $1 \sigma^{7}$ im Propsteigarten in Tütz in Westpreufsen.

Nach Kayser ist der Plattmönch bei Lissa die häufigste Grasmückenart; sie ist jetzt auch bei Posen recht häufig, während sie nach A. v. Homeyer 1866 hier ein ziemlich seltener Brutvogel war.

\section{Phylloscopus sibilator Bchst. - Waldlaubsänger.}

1912: Am 22. IV. liefs I Stück djü-Rufe an der Wartheseite des Kernwerks hören; am 27. IV. sang ein $\sigma^{7}$ im Kobylepoler Grunde; am 28. im Eichwalde. Am 2. V. sangen im Kobylepoler Grunde mindestens $3-4$ o

Am 4. VI. fand ich im Eichwalde ein Nest, in dem gerade die Jungen auskamen; neben 4 Eiern lagen 2 nackte Vögelchen. Als ich am 13. VI. das Nest besichtigte, lief's 1 Junges einen Angstruf hören; das alte $\sigma^{T}$ sang in der Nähe. Am 15. salsen die ausgeflogenen Jungen, 4 an der Zahl, unweit des Nestes etwa $21 / 2 \mathrm{~m}$ hoch auf einem Zweige. Sie wurden von dem $\$$ eiligst fortgeführt. Die Jungen folgten, nur eins verweilte etwas länger, ehe es sich zur Flucht entschlors. Währenddem sang das alte o. Im Neste lagen 2 unbefruchtete Eier.

1913: Am 25. IV. gegen Abend djü-Rufe oberhalb des Kobylepoler Grundes zu hören, dann auch mehrmals der Gesang.

Am 29. V. hörte ich daselbst fast nur das trillernde Sirrr oder Psrrr ohne die einleitenden Töne.

1914: Am 30. IV. singend und djü rufend im Solatscher Wäldchen (Oberlehrer Fabra).

1915: Am 21. IV. $1 \sigma^{x}$ singend in den Festungsanlagen vor dem Warschauer Tor; am 30. IV. in den Anlagen des Kernwerks reichlicher als sonst.

Nach Kayser bei Lissa ziemlich zahlreich.

\section{Phylloscopus trochilus L. - Fitislaubsänger.}

1912: Am 19. IV: sang 1 đa, durch das Kiefernwäldchen unterhalb der Militärfähre gegen den scharfen Ostwind gedeckt, im Weidengebüsch des rechten Wartheufers, 1 oder 2 ander auf der nahen Insel; am 24. überall reichlich.

Am 6. VIII. liefs 1 Stück um 6 Uhr p. am Steilufer der Warthe Gesang hören, ebendort auch um $6^{30}, 1 / 87$ und um \% 7 p. Da der Gesang etwas unfertig klang, schrieb ich ihn einem Jungvogel zu; auch am 9. VIII. versuchte sich daselbst 1 Stück im Gesange. 
1913: Die warme Witterung am Ausgang des März und Anfang des April hat den Rückzug dieser Art stark beeinflufst (auch bei andern Vögeln, z. B. Dorfschwalben, wurde dies bemerkt). Schon am 6. IV. hörte ich mehrmals die Strophe des Fitis im Kobylepoler Grunde (Sträucher belauben sich, Birken ergrünen, Schwarzdorn und Weichselkirsche blühen). An demselben Tage hörte Schulz den Fitis im Eichwalde. Die grofse Masse traf erst später ein: Am 21. IV, reichlich im Kobylepoler Grunde; am 24. IV. wimmelte es von Vögeln dieser Art an der Wartheinsel.

Die früh eingetroffenen Vögel sind offenbar auch früh zur Brut geschritten. So machte denn bereits am 20. VI. ein Jungvogel an der Wartheseite des Kernwerks Gesangsübungen, was ich sonst immer erst viel später beobachtet habe. Am 9. VIII. Gesangsübungen eines Jungvogels auf der Insel; am 27. VIII. versuchte 1 Stück seine Strophe im Weidicht des rechten Wartheufers gegenüber der Gärtnerei von Zippel.

1914: Am 15. IV. reichlich am Steilufer der Warthe und auf der Insel. Die Vögel dürften in den warmen Tagen vor den Osterfeiertagen eingetroffen sein, an denen meist stidliche und südwestliche Winde herrschten; am 16. IV. an der Nordseite des Kernwerks.

Am 4. VII. noch eifriger Gesang dieser Art am Steilufer der Warthe. Am 1. VIII. wurde wieder der Gesang junger Vögel im Weidicht der Insel gehört.

1915: Am 18. IV. erster Gesang an der Westseite des „Rohrteichs" (unfreundliche und rauhe Witterung).

Nach Kayser bei Lissa häufig.

\section{Phylloscopus collybita Vieill. - Weidenlaubsänger.}

1912: Die mehrere Wochen andauernde stürmische Witterung ausgangs März und in der ersten Hälfte des April verzögerte das Eintreffen dieser Art oder hemmte die gesangliche Betätigung. Dafür liefsen am 16. IV. nach Eintritt milderer Witterung gleich mehrere ơ ihren Gesang hören; im Eichwalde konnten 6-8 Stücke beobachtet werden.

Herbstgesang wurde, wie früher, häufig von dieser Art vernommen: Am 9. IX. 1 Stück singend an der Südseite des Kernwerks trotz rauher Witterung; am 17. IX. singend auf dem Schulhofe des Marien-Gymnasiums bei Regenwetter; ebendort singend am 18. und 23. IX. vormittags bei bedecktem Himmel und zeitweiligem Regen.

1913: Am 30. III. trieb sich um 1/410 a. 1 Stück neben einem Blaukehlchen in Weidenbüschen diesseit der städtischen Flufsbadeanstalt umher und liefs auch einmal einige leise Zil zel - hören (recht windig aus S. oder SO.); am 31. III. sangen im Eichwalde 5-6 Stücke (recht warm bei $\mathrm{S}$.). 
Herbstgesang wurde in diesem Jahre nicht vernommen. Die ungünstige Witterung im letzten Drittel des September und im Anfang Oktober hat die Vögel früher als sonst von hinnen getrieben.

Rittertor.

1914: Am 3. IV. Gesang auf den Kirchhöfen vor dem

Herbstgesang: Am 24. IX: auf dem Petrikirchhofe singend; am 25. IX. mittags singend auf dem Schulhofe des MarienGymnasiums. Weigold erwähnt im J. f. 0. 1911, S. 165 neben den gewöhnlichen huid-Rufen des Weidenlaubvogels einen Ruf djië; auch ich habe 1907 diesen absinkenden Ruf von einem Stück gehört und mit zíeb umschrieben (I, S. 577).

Nach Kayser bei Lissa häufig.

196. Regulus regulus L. - Gelbköpfiges Goldhähnchen.

Im Frühjahr nicht selten in Gärten und Parks, so am 23. III. 1912 vormittags mehrere im Vorgarten des MarienGymnasiums und am 17. IV. 1913 mehrere im Schillerpark; auch am 18. noch dort.

Im Spätherbst erscheinen die Vögelchen wieder an den genannten Stellen, besonders im November und Dezember: Am 2. XI. 1912 und am 13. XI. im Schillerpark unter Meisen; ebendort am 27. XI. 1913; am 9. XII. 2 Stücke auf den Linden am Schlofs trotz des grofsen hier herrschenden Verkehrs. Auch im Winter wurden mehrfach Goldhähnchen beobachtet: Am 22. XII. 1913 einige Stücke auf den niedrigen Fichten in den Solatscher Anlagen; am 28. XII. im Eichwalde; am 15. I. 19142 Stücke im Schillerpark; am 19. II. in der Nähe des Bismarck-Denkmals (ziemlich mild, Westwinde).

\section{Regulus ignicapillus [Brehm] Tem. - Feuerköpfiges Goldhähnchen.}

Am 17. X. 1863 beobachtete A. v. Homeyer zahlreiche Stücke auf dem Durchzuge. Mir ist nur einmal 1 Stück dieser Art zu Gesicht gekommen. Am 16, IV. 1911 trieb sich ein Feuerköpfiges Goldhähnchen ohne Scheu stundenlang in Fliederund Johannisbeersträuchern des Propsteigartens in Falkenau (Kr. Gnesen) umber (vgl. II.).

Nach Dr. Hesse (a. a. O., S. 601) ein Nest dieser Art im Berliner Museum: Nest; ohne Katalognummer. 8. VI. 1900. Wierzowka bei Posen. v. Treskow.

198. Hippolais icterina Vieill. - Gartensänger.

1912: Am 9. V. um $4^{10} \mathrm{Uhr}$ p. liefs sich der erste Gartensänger an der Wartheseite des Kernwerks vernehmen; der Vogel begann seinen Gesang mit mehreren Pirolrufen. Sonst war kein 
Stück zu hören, was bei der ungünstigen Witterung nicht auffällig war. Auch am 10. V. war nur dieser Vogel im Kernwerk zu hören; am 12. V. wurden bei einem Ausfluge nach Kobylepole an 2 Stellen singende $\sigma^{x}$ gehört und am 13. V. 2 oder 3 an der Wartheseite des Kernwerks, die bei der windigen und kühlen Witterung wenig sangeslustig waren. Die Gartensänger, in früheren Jahren hier fast gemein (vgl. I. und II.), waren wie im vorigen so auch in diesem Jahre fast selten zu nennen.

Mit Jungen umherziehende Vögel wurden am 6 . VIII. im Schilling, am 7. VIII. im Eichwalde, am 9. VIII. an der Wartheseite des Kernwerks, am 10. VIII. im Weidicht an der Scheibenseite der Schiefsstände vor dem Warschauer Tor und im Kobylepoler Grunde wahrgenommen, endlich noch am 24. VIII. eine Familie auf Wegbäumen am Gutshofe in Naramowitz; die Jungen wurden gefüttert.

1913: Am 12. V. 1 Stück an der Südseite des Kernwerks gegenüber dem Bahnhof Gerberdamm; etwas weiter ein 2. Stück.

In diesem Jahre war dieser treffliche Sänger hier wieder etwas reichlicher vertreten: Am 16. V. sangen an der Wartheseite des Kernwerks um 1/,12 a: 5 ð, an der Südseite des Kernwerks mindestens $7 \sigma^{x}$. Auf einem Gange durch den Eichwald hörte ich am 27. V. 16 singende $\sigma^{\top}$. Am 30. V. flocht 1 Stück im Eichwalde die einleitenden Töne aus dem Gesange des Trauerfliegenschnäppers in seinen Gesang ein.

Am 2. VII. und 4. VII. sangen noch mehrere $\sigma^{\star}$ recht eifrig in den prächtigen Gärten des Städtchens Powidz.

1914: Am 6. V. ein singendes $\sigma^{x}$ an der Wartheseite des Kernwerks; am 17. V. in den Festungsanlagen am "Städtchen“ bis 5 singende $\sigma^{x}$.

Am 2. VI. traf ich den Gartensänger bei Neudorf-Mauche, ferner bei Starkowo und bei Blotnik (Kr. Bomst); am 3. VII. singend in Heidedombrowka und in Lippe (Kr. Obornik); am 6. VII. singend in Antonin und am 7. VII. am Bahnhof Przygodzice, ein weiteres Stück in einem Garten von Dembnica (Kr. Adelnau).

Nach Kayser bei Lissa häufig; 1 Stück trug das Wäd wäd der Dorngrasmücke täuschend vor.

\section{Acrocephalus arundinaceus L. - Rohrdrossel.}

1912: Am 2. V. sang $1 \sigma^{x}$ am Schlofspark Ostend, am 7. V. 1 Stück im Weidicht des alten Warthebettes und ein anderes gegen Abend im „Rohrteich“. Reichlicher waren die Vögel erst seit dem 9. vertreten.

Am 29. VI. hörte ich noch eifrigen Gesang bei Ketsch, während sich die Vögel in der näheren Umgebung Posens schon still verhielten.

1913: Am 27. IV. liefs 1 Stück um 7 Uhr p. bruchstückweisen Gesang im alten Röhricht am Ostendpark hören; am 
28. IV. mehrere am kleinen Ketscher See singend; am 9. V. sangen zahlreiche Vögel im alten Röhricht am Schwersenzer See unweit der Zieliniec-Mühle. Die Vögel hatten sich hierher gezogen, weil sonst das Rohr abgeerntet war. Wo Weidenbüsche in der Nähe des Sees standen, boten diese den Rohrdrosseln erwünschten Unterschlupf. Am 7. V. fand ich diese Art reichlich am See von Kowalskie.

1914: Schon am 24. IV. sang kurz nach 6 Uhr p. $1 \sigma^{7}$ mit verhaltener Stimme im Weidicht unweit der Cybinabadeanstalt; am 30. V. sehr zahlreich am Schwersenzer See.

\section{Acrocephalus streperus Vieill. - Teichrohrsänger.}

1912: Am 12. V. um $6^{10} \mathrm{Uhr}$ p. hörte ich ein Stück im Kobylepoler Grunde und zwar im Buschwerk am kleinen Tümpel; am 14. sang 1 Stück im Rohrtümpel diesseit der Wartheinsel, und auch am Steilufer der Warthe war an 2 Stellen je ein o zu hören.

Noch am 6. VIII. hörte ich um 1/s $7 \mathrm{p}$. von dieser Art einige Touren an der Wartheinsel.

1913: Am 7. V. liefs 1 Stück gegen $3 / 47$ p. im Weidicht am „Rohrteich" bruchstückweisen Gesang hören; am 12. V. sangen daselbst $2 \sigma^{\top}$.

1914: Am 17. V. 1 Stück eifrig singend im alten Rohr eines Tümpels gegenüber Ostendpark; am 18. V. im „Rohrteich" singend, am 24. V. daselbst $2 \sigma^{\top}$; am 30. V. mehrere am Schwersenzer See.

Am 10. VI. sangen $2 \sigma^{T} \mathrm{im} R o h r$ des kleinen Teiches der Wolfsmühle, mehrere an der Insel, zum Teil im Weidicht.

\section{Acrocephalus palustris Bchst. - Sumpfrohrsänger.}

1912: Am 14. V. um 5 8/ p. eifriger Gesang dieser Art im Weidengebüsch der Warthe gegenüber der Gärtnerei von Zippel; am 17. V. um $3 \frac{1}{\mathrm{~s}} \mathrm{p}$. in der Nähe des städtischen Flufsbades. Reichlicher vertreten erst vom 20. an; an diesem Tage sang $1 \sigma^{r}$ in Syringensträuchern im Schilling, ein $\mathrm{zweites}$ ebendort in einer Teufelszwirnhecke.

In der zweiten Hälfte des Juni liefs der Sangeseifer der hiesigen Vögel merklich nach; am 29. VI. hörte ich noch 1 Stück am grofsen Ketscher See, es ahmte streperus und schoenobaenus sehr gut nach.

1913: Am 17. V. 1 o $^{\text {T }}$ singend im Gluwnatale bei Jerzykowo; am 18. V. unterhalb des Schillings bis zur Militärfähre mindestens $30^{x}$.

Am 31. V. reichlich an der Warthe ober- und unterhalb der Militärfähre und an der Insel. Ein $\sigma^{\star}$ liefs den Ruf des Wendehalses und das wihd $\mathrm{k}$ der Nachtigall deutlich hören. 
Noch am 4. VII. hörte ich gegen Abend in Falkenau (Kr. Gnesen) auf einem mit Gemenge bestandenen Ackerstücke 2 eifrig singende $\sigma^{x}$; Weidengebüsch war weit und breit nicht vorhanden.

1914: Am 17. V. ein eifrig singendes $\sigma^{x}$ im Weidengebüsch eines Grabens diesseit Johannistal. Bei Posen in diesem Jahre wieder recht reichlich vertreten. Am 30. V. $2 o^{\top}$ am Schwersenzer See.

Am 12. VI. gab ein $\sigma^{\top}$ gegen 8 Uhr p. unweit von Johannistal deutlich Rufe der Kohlmeise und das Liedchen des Fitislaubvogels wieder. Am 15. VI. sang 1 Stück in einem Roggenfelde diesseit der Solatscher Teichanlagen. Am 1. VII. 1 singendes Stück in einem Haferfelde an der Bogdanka zwischen dem Ziegelwege und den Solatscher Anlagen.

Kayser hörte 3 Stücke unweit von Lissa im Erlenbusch und im Priebischer Bruch.

\section{Acrocephalus schoenobaenus L. - Schilfrohrsänger.}

1912: Am 24. IV. sang $1 \sigma^{x}$ auf einer der kleinen schwimmenden Inseln am Schlofspark Ostend; vom 27. ab waren Schilfrohrsänger reichlicher vertreten.

Am 23. VI. sang $1 \sigma^{x} \mathrm{im}$ Gemenge von Sommerroggen und Wicken unweit der Zieliniec-Mühle; auch Balzflug wurde fleifsig geübt. Ebenso machte es 1 Stück am 26. VI. im Sumpfschachtelhalm diesseit der Wartheinsel. Am 27. VI. sang $1 \sigma^{\top}$ in einem Roggenstücke jenseit der Kläranlage. In diesen Fällen handelte es sich offenbar um Ersatz- oder zweite Bruten.

1913: Am 25. IV. singend am Schwersenzer See und im Kobylepoler Grunde.

1914: Am 26. IV. sang $1 \sigma^{\pi}$ im Weidicht der alten Warthe einige Touren; am 29. IV. 1 Stück in dem kleinen Erlengebüsch auf den Wiesen bei Johannistal. Bruchweifskehlchen waren in diesem Jahre bei Posen weniger häufig als sonst.

Schon A. v. Homeyer nannte diese Art 1864 bei Posen äufserst gemein, ebenso bei Mixstadt (Kr. Schildberg).

\section{Acrocephalus aquaticus $\mathrm{Gm}$. - Binsenrohrsänger.}

Im neuen Naumann (II, S. 44) wird der Binsenrohrsänger als Bewohner der Provinz Posen aufgeführt, offenbar auf Grund einer Angabe A. v. Homeyers, der am 22.-25. IX. 1863 diese Art bei Smielow (welches?) beobachtet hat (Schulz a. a. 0., S. 77; vgl. auch Borggreve a. a. O., S. 92): A. v. Homeyer fand Salicaria cariceti an der polnischen Grenze. Danach dürfte es sich um den im Kreise Ostrowo liegenden Ort handeln.

Dr. Hesse führt (a. a. 0., S. 602) ein Nest dieser Art aus dem Berliner Museum an, das von A. v. Homeyer eingeliefert worden ist, jedoch ohne nähere Angabe des Fundortes: Nest. Ohne Katalognummer. Mai 1864. Posen. A. v. Homeyer. 


\section{Locustella naevia Bodd. - Heuschreckensänger.}

1912: Schon am 24. IV. sirrte ein Vogel eifrig im Kobylepoler Grunde am rechten Ufer der Cybina an einer mit spärlichem Rohr und niedrigen Erlenbüschen bestandenen Stelle. Trotzdem ich dem Vogel ziemlich nahe war, konnte ich ihn nicht erspähen. Nur wenige Tage später, am 27. um $5 \frac{1}{s}$ p., machte sich $1 o^{\text {r }}$ gegenüber Schlofspark Ostend bemerkbar. Beide Vögel wurden auch in der Folgezeit an den genannten Stellen oder in der Nähe derselben angetroffen und zwar das Stück im Kobylepoler Grunde am 2. V. um $61 / \mathrm{c}$. und am 5. und am 17. V., das Stück gegenüber Schlorspark Ostend am 21. VI. um 6 Uhr p.

Recht spät zog dagegen 1 Stück, das am 17 . V. um $31 / 3$ p. in der Nähe des städtischen Flufsbades vor dem ehemaligen Eichwaldtore sang. In diesem Falle handelte es sich sicher um ein durchziehendes Stück, da die Stelle zum Brüten wegen des grofsen Menschenverkehrs kaum geeignet ist. Der Vogel wurde hier denn auch nicht mehr angetroffen. Aufserdem traf ich den Schwirl auch wieder am Steilufer der Warthe (vgl. I. und II.) und auf der nahen Wartheinsel, wo er nun schon mehrere Jahre hindurch während der Brutzeit verweilt hat. Am 7. V. sang um 4\% p. 1 Stück am Steilufer und ebendort um $7 \mathrm{Uhr}$ p.; auch am 9., 11., 12. und 15. V., am 7., 12. und 17. VI. ebendort. Von da ab hörte ich den Vogel auf der Insel und zwar am 26., 27. und 28. VI. meist gegen $7 \mathrm{Uhr}$ p.

Ferner war am 17. V. um $71 / 4$ p. 1 Stück auf den mit Buschwerk durchsetzten Wiesen diesseit der Loncz-Mühle zu hören, auf das ich schon einige Tage vorher aufmerksam geworden war.

Danach war der Heuschreckensänger im Jahre 1912 in der näheren Umgebung Posens keineswegs selten, eher verhältnismäfsig häufig vertreten. Dafs es sich dabei zum Teil um Brutvögel gehandelt hat, ist $\mathrm{m}$. E. zweifellos.

1913: In diesem Jahre fehlte auf der Wartheinsel diese Art. Offenbar hatte die zur Unzeit eintretende Überschwemmung (Ausgangs April und erste Hälfte des Mai) die Vögel vertrieben oder ihre Ansiedelung unmöglich gemacht.

1914: Am 23. V. liefs 1 of kurz nach 5 Uhr p. auf der Insel einige kurze Schwirrtouren hören. Auch in der Folgezeit wurde hier mehrmals mindestens 1 sirrendes $\sigma^{x}$ gehört, so am 10. VI. um $1 / 28$ p., am 13. VI. gegen $1 / 28$ p. und am 24 . VI. gegen 7 Uhr $\mathrm{p}$.

Nach Schulz wurde diese Art schon 1864 von A. v. Homeyer bei Posen festgestellt.

Kayser hörte am 26. V. 1914 vormittags zwischen 6 und 10 Uhr $2 \sigma^{\pi}$ im Priebischer Bruch. Am 14. V. 1915 schwirrten nach demselben im Tharlanger Bruch 3 ơ, am 1. VI. 1915 im Priebischer Bruch ebenfalls etwa 3. 
205. Locustella fuviatilis Wolf. - Flufsrohrsänger.

Der Schlagschwirl wurde zum ersten Male für die Umgebung von Posen, sowie für die Provinz am 18. V. 1906 in einer Lichtung am Nordrande des Eichwaldes festgestellt (vgl. 0. Mb. 1908, Maiheft). Über das Auftreten dieser Art bei Posen in den folgenden Jahren (vgl. I. und II.).

1912: Am 12. V. begann um $5^{\text {br }} \mathrm{Uhr}$ p. ein Flufsrohrsänger im Kobylepoler Grunde am rechten Cybinaufer zu singen und zwar anscheinend in einem Weidenbusche, kaum 20-30 Schritte von einem eifrig sirrenden naevia- $\sigma^{x}$ entfernt. Der Vogel sang ziemlich lange Touren. Der Lautkomplex sätte - - (oder sette) schien mir immer noch am besten den Gesang von fluviatilis wiederzugeben.

Am 15. V. liefs sich 1 Stück um $5^{10} \mathrm{Uhr}$ p. an der Cybina gegenüber Schlofspark Ostend vernehmen. Vielleicht war dieser Vogel mit dem vorher genannten identisch; jedenfalls wurde an der früher bezeichneten Stelle kein Vogel mehr beobachtet.

Am 31. V. sang 1 Stück im Eichwalde um $6^{50} \mathrm{Uhr}$ p. in einer kleinen Lichtung in den hohen Eichen südlich des Bahndammes. Daselbst hörte ich den Vogel auch am 8 . VI. um $1 / 28$ p., dann jedoch nicht wieder.

Am 29. VI. traf ich $1 \sigma^{\top}$ um $7 \frac{1}{2}$ p. im Bogdankatale unweit der Elsenmühle.

Im Jahre 1913 wurde kein Flufsrohrsänger bei Posen wahrgenommen.

1914: Am 7. VI. sang um $1 / 46$ p. $10^{7}$ eifrig im grasdurchwachsenen Weidicht des alten Warthebettes oberhalb der Wolfsmühle. Auch in der Folgezeit wurde der Schlagschwirl hier mehrfach beobachtet, so am 10 . VI. gegen $8 / 6$ p., am 13. VI. um $1 / 8$ p., am 16. VI. um 7 Uhr p. An dem zuletzt genannten Tage hörte ich bald nach $1 /, 8 \mathrm{Uhr}$ auch an der Cybinamündung 1 Stück. Es wurde später hier nicht mehr vorgefunden.

Am 26. V. 1914 hörte Kayser im Priebischer Bruch ,eine Anzahl Männchen" etwa zwischen 6 und 10 Uhr fleifsig singen; am 1. VI. 1915 sangen etwa $3 \sigma^{x}$ im Priebischer Bruch. Ein $\sigma^{x}$ sang sogar im Erlenbusch, dicht an der Stadt Lissa. Die voranstehenden Beobachtungen lassen auf das Brüten dieser Art im Posenschen mit Sicherheit schliefsen.

\section{Iroglodytes troglodytes L. - Zaunkönig.}

Am 26. I. 1913 liefs um $8 / 612$ a. 1 Stück mehrmals auf einem Kirchhofe am Schillerpark seinen Gesang hören (heftiger, böiger Wind aus W., doch ab und zu ein Sonnenblick; das Thermometer zeigte etwas über $0 \mathrm{Grad}$; auch die Meisen sind recht fröhlich und ein Grünling macht Gesangsstudien).

Herbstgesang hörte ich von 1 Stück am 21. X. um $8 / 44$ p. in der Wolfsmühle. 


\section{Turdus musicus L. - Singdrossel.}

1912: Am 5. III. mehrfach schreckend zwischen Bromberger und Kalischer Tor, am 18. III. daselbst singend.

Am 11. VI. fand ich ein Drosselnest mit 3 Eiern. Das Nest, stark nach einer Seite geneigt, war verlassen. Es war das vermutlich durch die Einwirkung des gewaltigen Regengusses vom 10. VI., der besonders zahlreiche Nester von Grasmücken vernichtet hatte, geschehen.

Am 19. VI. bemühte sich $1 \sigma^{x}$ im Eichwalde Nachtigallstrophen wiederzugeben, wobei es sich jedoch von dem Rhythmus des Drosselliedes nicht recht losmachen konnte.

1913: Am 17. III. hörte ich im Kernwerk den ersten Gesang.

1914: Am 17. III. erster Gesang in den Anlagen vor dem Warschauer Tor.

\section{Turdus iliacus - Weindrossel.}

1912: Am 22. III. traf ich auf den Eichen im südlichen Teile des Eichwaldes einen lebhaft schwatzenden Schwarm, aus dem auch hin und wieder die Heultour (tjyr - _ _ - ) oder Bruchstücke derselben zu hören waren. Am 26. III. ebendort unter Wachholderdrosseln. Die Heultour von iliacus, die heute wie dji - - - klang, sowie das Schackern von pilaris war mehrfach zu hören. Auch am 15. IV. wurden noch Weindrosseln an der Südseite des Kernwerks und am 16. IV. an der Nordseite des Eichwaldes und südlich des Eisenbahndammes beobachtet, unter denen einige Wachholderdrosseln sichtbar waren. Wahrscheinlich hat die bis zum 15. herrschende stürmische Witterung die Vögel aufgehalten; ja selbst am 20. IV. wurden noch an 2 Stellen im Eichwalde und am 21. vor dem Warschauer Tor Weindrosseln gehört, die sich durch Bruchstücke der Heultour kenntlich machten, und am 24. 1V. trieb sich ein Flug von 13 Stücken im Kobylepoler Grunde umher, aus dem quip-Rufe und ein leises Geschwätz zu vernehmen waren.

Auf dem Durchzuge im Herbst sah ich am 7. XI. kurz nach 1/. 4 Uhr p. 1 Stück im Schillerparke, das recht vertraut war, und am 19. XI. hörte ich quip- oder kip-Rufe von ziehenden Drosseln, die offenbar dieser Art angehörten, im Eichwalde. Die Vögel safsen zerstreut im Geäst der hohen Bäume.

1913: Am 23. III. sah ich 4 Stücke auf einer Birke in dem lichteren Teile des Kiefernwäldchens jenseit Kobylepole. Dio Vögel flogen unter kip-Rufen den Kiefern zu, wo ein gröfserer Schwarm das bekannte Geschwätz vollführte, aus dem sich ab und zu Bruchstücke der Heultour (dji - - ) herauslösten. Ähnlich betätigte sich am 6. IV. ein kleiner Schwarm im Kobylepoler Grunde. Am 14. und 15. IV. traf ich einen ziemlich starken 
Schwarm am Lubascher See, am 18. IV. einen Schwarm im Eichwalde südlich des Bahndammes. Am 25. IV. wurde noch mehrmals die Heultour im Kobylepoler Grunde gehört.

1915: Am 8. IV. zahlreich, mit Wachholderdrosseln gemischt, am See von Lubasch, schwatzend und Bruchstücke der Heultour hören lassend.

\section{Turdus viscivorus L. - Missteldrossel.}

Kayser (a. a. O., S. 20) berichtet über ein am 7. X. 1912 am Telegraphendraht erstofsenes Stück; diese Drossel ist bei Lissa nur Durchzügler.

Bei Posen wurde diese Art nur selten auf dem Zuge angetroffen; in den Kiefernwäldern bei Schönlanke ist sie Brutvogel (Schulz). An der Prosna ist sie von A. v. Homeyer beobachtet worden.

\section{Turdus pilaris L. - Wachholderdrossel.}

1912: Umherstreichende und teilweise wohl hier überwinternde Wachholderdrosseln wurden mehrfach angetroffen. Am 1. II. traf ich einen starken Schwarm am Steilufer der Warthe, über das Buschwerk zerstreut; am 7. II. ein Paar ebendort, lebhaft warnend, ein drittes Stück flog schackernd ab. Am 9. III. traf ich ein einzelnes recht vertrautes Stück auf den Wiesen an der Cybinamündung; am 25. 3 Stücke an den Wiesen bei Gluwno, andere abends zwischen Bromberger und Warschauer Tor, lebhaft schwatzend. Noch am 18. IV. flogen um $41 \%$ p. von den Erlen an der Cybinamündung 13 Stücke unter lebhaftem Schackern ab.

Auf dem Herbstzuge: Am 30. XI. 4 Stücke im Eichwalde; ebendort war am 17. XII. das Schackern dieser Art zu hören.

1913: Am 17. I. um 1/,4 p. ein ziemlich starker Schwarm auf den Wiesen diesseit der Insel, in ihrer Nähe zahlreiche Nebelkrähen; 2 pilaris flogen schackernd ab. Auch im Buschwerk des Steilufers trieben sich einige umher. Am 24. II. auf den Wiesen in der Nähe der Cybinamündung ein einzelnes Stück, am 4. III. wieder ein Stück an derselben Stelle, vielleicht derselbe Vogel.

Am 27. XI. traf ich ein einzelnes Stück im Schillerpark, bald an der Erde, bald auf Büschen. Am 29. war der Vogel noch dort, er sals ruhig im Gezweige einer Weide. Am 7. XII. sah ich 1 Stück auf dichtem Schwarzdorngebüsch zwischen Kläranlage und Steilufer. Nach dem Verhalten (recht vertraut, neugierig) möchte ich annehmen, dafs es der vorher im Schillerpark beobachtete Vogel war, der dort am 30. nicht mehr vorgefunden wurde. Der Vogel war offenbar verletzt.

1914: Am 8. II. 4 Stücke auf den Wiesen jenseit der städtischen Anlagen am Vicktoriapark auf der Nahrungssuche. 


\section{Turdus merula L. - Amsel.}

Überwinternde oder zur Winterszeit durchziehende Stücke im Jahre 1912: Am 7. I. 1 Stück im Schilling; es flog um 4 Uhr dem Buschwerk an der Cybinamündung $z u ;$ am 1. II. 1 Stück am Steilufer der Warthe, schreckend; am 5. II. 1 Stück im Schilling (es ist wohl das unter 7. I. genannte Stück; Schneedecke, nachts $-15^{\circ}$ ); am 7. II. wurde auch das Stück am Steilufer wieder geseben und gehört, ebenso am 17. II. Am 1. III. gegen Abend 2 Stücke an der Wartheseite des Kernwerks, offenbar schon Rückzügler.

Am 6. III. gegen Sonnenuntergang Amselgesang gegenüber Schlofspark Ostend und am 7. III. um dieselbe Zeit an der Cybinamündung. Von nun an überall in der Umgebung Posens, auch auf einem der Kirchhöfe am Schillerpark und auf dem Petrikirchhofe; sogar im Buschwerk des kleinen Gartens der Wolfsmühle sang $1 \sigma^{x}$ sehr eifrig.

Im Schillerpark und auf den anstofsenden Kirchhöfen trieben sich Amseln im Herbste recht lange umher: Am 30. X. 1 Stück im Schillerpark, am 1. XI. daselbst 3 Stücke (Prof. Dr. Gerigk); am 6. und 7. XI. befehdeten sich daselbst 2 Stücke mit ärgerlichem Tik - - oder Tix _ - - wobei es den Anschein hatte, als ob 1 Stück das andere aus dem Revier zu vertreiben suchte. Das schien gelungen zu sein; denn am 13. XI. war 1 Stück auf dem Petrikirchhofe sichtbar, während im Schillerpark seitdem nur 1 Vogel zu sehen war. Am 30. XI. war der Schreckruf der Amsel im Eichwalde zu hören und am 6. XII. daselbst ein Stück sichtbar, das sich auch am 17. XII. bemerkbar machte und auch am 20. XII. sein leises Duck hören liefs.

1913: Ein im Schillerpark überwinterndes Stück wurde mehrmals im Januar und Februar und im Anfang März beobachtet.

Erster Gesang am 10. III. gegen 5 Uhr p. auf dem GarnisonFriedhof (westliche Winde mit Regen- und Schneeböen).

Am 18. VI. flocht im Eichwalde eine Amsel in ihren Gesang ab und zu das Klüh des Schwarzspechts ein. Es wurde stets ans Ende einer Strophe gestellt. Die Tonfärbung wurde so genau getroffen, dafs ich zuerst immer nach einem Schwarzspecht als dem vermeintlichen Urheber Umschau hielt, bis ich endlich eine Amsel als Urheberin dieses Tones mit Sicherheit feststellen konnte. Schwarzspechte gab es zudem jetzt hier nicht mehr. Offenbar mufs die Amsel den Winter hier verbracht und dabei eine Vorliebe für diesen Spechtruf gefalst haben. In der Tat habe ich an der Stelle mehrfach während des Winters ein Amsel- $\sigma^{\top}$ angetroffen.

Am 12. XI. 1 Stück im Schillerpark sichtbar; am 23. gegen Abend daselbst 3 Stücke, von denen 2 sich kräftig befehden. Am 5. XII. 2 Stücke sichtbar, einträchtig auf einer Weide sitzend, ebenso am 7. XII., offenbar ein Pärchen, das auch im Winter 
getreálich zusammenhielt. Am 7. XII. auch 1 Stück am Steilufer der Warthe, mit dak warnend. Am 9. XII. ein junges $\sigma^{x}$ oder $\bigcirc$ im Schlofsgarten auf einer Eberesche (Schneedecke). Am 20. XII. das Pärchen im Schillerpark um $1 / 24$ Uhr deutlich sichtbar. Während das $Q$ in einem Weidenbusche am Teiche sals, lief das $\sigma^{x}$ am Rande des Teiches umher, ging dann in das seichte Wasser und nahm ein Bad, wobei es sich klatschnafs machte. Da kam das $\%$ heran und griff das $\sigma^{\top}$ an, das denn auch das Wasser verliefs und auswich. Das \& wollte vermutlich den unvorsichtigen Gatten vor den üblen Folgen des unzeitgemäfsen Bades bewahren (das Thermometer zeigte um Null herum). Am 27. XII. war das Pärchen wieder sichtbar, an verschiedenen Stellen unter den Sträuchern Nahrung suchend. An den im Schillerpark eingerichteten Futterstellen habe ich bisher keine Amsel gesehen.

1914: Das Pärchen im Schillerpark hielt auch im Januar hier den Unbilden der Witterung stand. Am 3. I. und 14. I. 1 Stück am „Rohrteich" sichtbar. Eintretende Schneefälle brachten Zuzug. Es handelte sich dabei offenbar um Stücke, die aus östlíchen Gegenden kamen und dem Schnee auszuweichen versuchten. So waren infolge des in der Nacht vom 30./31. Dezember herrschenden Schneetreibens an verschiedenen Stellen Amseln anzutreffen, die aber bald weiter rückten: 1 Paar in der Gärtnerei von Zippel; 1 Stück in einem Schwarzdorngebüsch jenseit der Kläranlage, von einer andern Drossel, anscheinend pilaris, von welcher Art ich dort vorher 1 Stück beobachtet hatte, angefeindet; 2 Stücke am Steilufer der Warthe schreckend und sichtbar.

Am 2. III. erster Amselgesang im Goethepark (Prof. Dr. Gerigk); 15. III. Gesang auf dem Petrikirchhofe; am 18. III. lebhaftes Amselkonzert im Eichwalde, an dem sich zahlreiche Stücke beteiligten.

Am 7. V. fand ich ein Nest an der Wartheseite des Kernwerks. Als die Jungen schon ziemlich herangewachsen waren, war eines Tages das Nest mit dem Inhalt verschwunden.

1915: Am 10. I. sang um $31 / \mathrm{s} .1 \sigma^{x}$ etwa 10 Minuten lang eifrig auf einer hohen Kastanie an der Wittingstrafse; es flog dann in den nahen Goethepark und liefs auch hier seinen Gesang hören. Das war bei uns bisher unerhört (Witterung schön, mittags sonnig bei SW., nachts leichter Frost); am 14. II. im Goethepark singend, wohl dasselbe Stück; ein anderes sang auf dem Kirchhofe an der Garnisonkirche (warm und sonnig). Es scheint eine Umbildung unsrer Park- zur Stadtamsel im Wege zu sein. Die erste Hälfte des März brachte sehr rauhe Witterung und starke Fröste (bis $13^{\circ}$ ). Dadurch war auch den Amseln das Singen verleidet. Erst am 14. III. um 9 Uhr a. hörte ich Gesang von einem $\sigma^{x}$ auf dem Petrikirchhofe. Noch am 15. VII. sang je ein $\sigma^{\top}$ eifrig auf dem Petrikirchhofe und im Eichwalde. 
Nach Kayser ist die Amsel bei Lissa häufig, in den Wäldern zahlreicher als die Singdrossel, in der Stadt als Gartenvogel eingebürgert.

212. Saxicola oenanthe L. - Steinschmätzer.

1912: Am 19. IV. begrülste mich knicksend 1 Stück auf dem Kohlenstapel am Bahnhof Gerberdamm; es wurde auch in der - Folgezeit hier angetroffen und hat sicher hier wieder gebrütet. Am 21. IV. sang $1 \sigma^{x}$ einige Strophen auf einem Wegbaume der militärischen Strafse jenseit der Schiefsstände vor dem Warschauer Tor und flog dann den benachbarten sandigen Feldern zu.

Auf dem Abzuge verweilen die Steinschmätzer gern auf umgepflügten Ackerstücken, so am 2. IX. 1 Stück auf der Feldmark von Kreising und am 12. IX. 1 Stück auf den umgestürzten Sandflächen jenseit der Loncz-Mühle.

1913: Am 6. IV. liefs 1 Stück auf einem Steinhaufen an der städtischen Flufsbadeanstalt eine Strophe hören. Der Steinschmätzer ist hier Brutvogel. Auch 1910 hat in diesem Steinbaufen 1 Pärchen Junge erbrütet, die ich mehrmals unweit der Stelle beobachtet habe (vgl. II.).

Am 10. V. traf ich in der Nähe des Bahnhofs Ketsch 1 Stück, das auf einem Telegraphendrahte fleifsig sang.

1914: Am 19. IV. trieb sich 1 Stück auf dem Platze der ehemaligen Cegielskischen Fabrik und auf dem durch die Strafse davon getrennten Holzlagerplatze umher.

Am 30. V. sah ich eiu Pärchen auf dem Gelände der Zementröbrenfabrik diesseit Kobylepole. Das Paar gebärdete sich sehr ängstlich; es hatte offenbar Junge.

1915: Auf dem Abzuge waren mehrere Stücke, die ich am 2. IX. bei Kreising auf Sturzacker sah.

\section{Pratincola rubetra L. - Braunkehliger}

Wiesenschmätzer.

1912: Am 1. V. sang 1 Stück um $4^{20} \mathrm{Uhr} p$. in der Nähe der Kläranlage, mehrere andere liefsen in der dichtbegrasten Schonung links des Weges von Naramowitz ihren Gesang hören (NW., nachts $-2^{\circ}$, dürr und staubig). Die ungünstige Witterung hat ihre Ankunft etwas verzögert, da sie bei günstigem Wetter schon in der zweiten Hälfte des April einzutreffen pflegen. Die Wiesenschmätzer waren in diesem Jahre bei Posen ziemlich reichlich vertreten.

Gesang wurde bis in das letzte Drittel des Juni vernommen: Am 23. VI. bei Johannistal und in der Nähe von Schwersenz; am 26. an der Kläranlage; am 27. ebendort. Hier ahmte um 1/28 p. ein eifrig singendes $\sigma^{\top}$ den Buchfinkenschlag (etwas gekürzt, aber der Rhythmus festgehalten) und die Strophe des Hausrotschwanzes nach und bemühte sich, den Pfiff des Pirols wiederzugeben. 
1913: Am 24. IV. 1 Stück gegen 5 Uhr p. im Weidicht unterhalb der Wolfsmühle deutlich sichtbar, doch still; am 25. 2-3 singende $\sigma^{x}$ am Schwersenzer See.

Am 7. V. traf ich in den Anlagen an der Oborniker Chaussee 6-7 $\sigma^{x}$, darunter auch gute Spötter. Ein Stück liefs den Buchfinkenschlag und anderes hören. Zahlreich waren die Vögel am 13. V. auf den Wiesen am Kostener Obra-Kanal bei Kurzagura, noch reichlicher am 14. V. im Obrabruch zwischen Gr. Sepno und Bonikowo an der Strafse und dem diese begleitenden Eisenbahndamme. Noch am 28. VI. hörte ich von $2 \sigma^{7}$ Gesang diesseit. der Wartheinsel.

Auf dem Abzuge: Am 1. IX. eine Familie unweit der Militärfähre und am 8. IX. einige Stücke in dem Rübenfelde an der Kläranlage. Später wurden keine mehr beobachtet.

1914: Am 21. IV. $1 \sigma^{\top}$ in der Nähe der Cybina auf den Wegbäumen der Schwersenzer Chaussee unweit der Jeliniec-Mühle singend und mehrmals ein haubenlerchenartiges Titüdüe hören lassend; am 22. IV. mehrere eifrig singend unterhalb der Wolfsmühle; am 29. IV. 3 singende $\sigma^{T}$ auf den Wiesen bei Johannistal.

Am 22. V. 1915 liefs $1 \sigma^{x}$ im Obrabruch bei Kosten in der Nähe der Brücke über den Moschiner Obra-Kanal mehrmals den Buchfinkenschlag hören.

Kayser hat diese Art bei Lissa öfter beobachtet. Am 7. VI. 1914 gab $1 \sigma^{\Upsilon}$ den Gesang der Dorngrasmücke täuschend wieder.

\section{Pratincola rubicola L. - Schwarzkehliger Wiesenschmätzer.}

Am 9. V. 1909 wurde ein schönes altes $\sigma^{\top}$ an der Rückseite der Schiefsstände vor dem Warschauer Tor beobachtet. Man vergleiche das Nähere in I. Anscheinend in der Gesellschaft des ${ }^{x}$ befanden sich 2 Vögel mit fahlbraunen Köpfen, die sehr vertraut taten. Vielleicht waren es 2 junge $\&$ dieser Art. Da ich bezüglich des $\sigma^{*}$ einen Irrtum für ausgeschlossen halte, nehme ich diese Art in meine Liste auf.

215. Cinclus aquaticus Bchst. - Wasserschmätzer.

Nach Schulz (a. a. O., S. 77) aus 2-4 Forstbezirken des Regierungsbezirks Posen 1907 als Brutvogel gemeldet. Die Angabe ist erfolgt auf Grund einer Umfrage des Geheimrats Conwentz aus dem Jahre 1907. Nähere Angaben wurden nicht gemacht, so dafs eine Nachprüfung unmöglich ist. Zwei Exemplare aus der Provinz befinden sich im Mielzynski-Museum. Fundort und Gewährsmann sind nicht genannt.

\section{Erithacus titys L. - Hausrotschwanz.}

1912: Während Schulz am 4.IV. in Schneidemühl die Strophe des Hausrotschwänzchens hörte, vernahm ich seinen Gesang bei 
Posen erst am 16. IV. (am 15. endlich der Wind zu leichtem NW. und dann 0 . ahgeflaut, nachdem 3 Wochen lang meist sehr starke Winde aus W., SW. und NW. geweht hatten, die Regen-, Graupelschauer und Schneeböen brachten; am 12. und 13. gab es richtige Wintertage mit heftigen Schneestürmen).

Gesang im Spätsommer und Herbst: Am 23. und 25. VIII. um $91 / 2$ a. auf der Bernhardinerkirche; am 30. VIII. an der Eisenbahnbrücke im Eichwalde; am 14. IX. um $1 / 29$ a. auf der Bernhardinerkirche; am 5. und 6. X. in Tütz (Westpreufsen).

1913: Am 6. IV. sang ein or (grau) in der Gartenwirtschaft Johannistal.

Am 29. VIII. um $3 / 43$ p. singend in Dembsen; am 1. IX. morgens an der Bernhardinerkirche singend; am 28. IX. gegen Sonnenuntergang im Schilling.

1914: Am 31. III. $1 \sigma^{x}$ singend auf einem der Holzlagerplätze vor dem ehemaligen Eichwaldtore; am 1. IV. gegen Abend am Steilufer der Warthe in der Nähe militärischer Gebäude.

Am 24. IX. und 11. X. Gesang bei Posen, am 3. X. in Lubasch.

Nach Kayser ist diese Art bei Lissa ziemlich spärlich.

217. Erithacus phoenicurus L. - Gartenrotschwanz.

1912: Am 22. IV. nachmittags singend an der Wartheseite des Kernwerks.

Letzter Gesang in der Brutzeit am 1. VII. an der Eichwaldstrafse in der Nähe des Viktoriaparks. Auffallender Weise sang am 26. VIII. kurz vor $9 \mathrm{Uhr}$ a. 1 Stück eifrig auf dem Petrikirchhofe; es war wahrscheinlich ein junges $\sigma^{x}$.

Am 12. IX. war 1 Stück an und auf der Umzäunung des Kirchhofs bei der Loncz-Mühle sichtbar. Später wurde kein ziehendes Stück mehr wahrgenommen.

1913: Am 17. IV. üm $\% 9$ a. eifriger Gesang eines or auf dem Petrikirchhofe; gegen 11 Uhr a. sangen 3 Stück an der Südseite des Kernwerks.

1914: Am 16. IV. 1 oder $2 \sigma^{x}$ singend auf dem Garnisonfriedhof; am 18. IV. im Eichwalde singend, am 19. IV. auf dem Petrikirchhofe.

1915: Am 23. IV. $1 \sigma^{\top}$ singend an der Südseite des Kernwerks, am 24. IV. auf einem Kirchhofe am Schillerpark.

Nach Kayser ist diese Art bei Lissa häufig.

218. Erithacus rubecula L. - Rotkehlchen.

1912: Am 26. III. an 5 oder 6 Stellen im Eichwalde singend (schwüler Lenztag mit Regenneigung, Westwind); Schulz sah schon am 24. 1 Stück im Eichwalde. Am 27. III. Gesang an der Wartheseite des Kernwerks (warm bei bedecktem Himmel, Südwest, abends gegen $8 \mathrm{Uhr}$ starkes Gewitter mit Regen; um $1 / 27$ p. flog eine Fledermaus über der Warthe hin und her). 
Zweite Gesangsperiode: Am 1. VI. sangen mehrere Stücke im Kobylepoler Grunde. Noch am 27. VI. Gesang abends an der Wartheseite des Kernwerks.

Der Abzug unserer Rotkehlchen beginnt wohl schon im September, ja vielleicht sind die Vögel, die sich dann bemerkbar machen, schon Durchzügler. Doch manche Stücke können sich von liebgewordenen Stellen nur schwer trennen. So zieht sich denn gerade bei dieser Art der Zug lange hin, bis in den Oktober und November: 30. X. zickernd im Schillerpark; ebendort am 13. XI. 2 Stücke. Beide Stücke liefsen, etwa 30 Schritt von einander entfernt, leisen Gesang hören, und es hatte den Anschein, als ob eins das andere aus dem erwählten Reviere vertreiben wollte. Am 19. XI. gegen $1 / 212$ a. machten sich beide Vögel durch Zickern bemerkbar und I Stück liefs mehrmals leisen Gesang hören. Am 26. XI. sang wieder $1 \sigma^{x}$ leise um $3 \mathrm{Uhr}$ p., ebenso am 5. XII. Das zweite Stück machte sich an einer entlegeneren Stelle durch Zickern bemerkbar. Auch am 10., 11. und 16. XII. wurden die Vögel hier wahrgenommen. Die zahlreichen Besucher der Eisbahn und die dadurch hervorgerufene Unruhe schienen dann den Vögeln den Platz verleidet zu haben. Am 17. XI. und am 16. XII. bemerkte ich auch im Goethepark ein Stück. Am 20. XII. sang gegen Sonnenuntergang $1 \sigma^{\top}$ ziemlich laut im Eichwalde unweit der Gastwirtschaft (sonnig bei SW.).

1913: Die vorher genannten beiden Vögel im Schillerpark machten sich wieder bemerkbar, überwinterten also offenbar daselbst, so am 2. I. (bisher recht milde Witterung, nachts leichter Frost), am 26. I. und am 30. I. Am letztgenannten Tage trieb sich 1 Stück an einer Futterstelle umher (nachts - 14\% ${ }^{\circ}$. Am 23. I. wurde auch das im Goethepark beobachtete Stück wieder gehört. Am 5. II. zickerte 1 Stück in den Anlagen an der Franziskanerkirche.

Am 20. III. erster Gesang gegen $6 \mathrm{Uhr}$ abends in den Anlagen vor dem Warschauer Tor, am 21. im Eichwalde. Rotkehlchen waren in diesem Jahre überall ziemlich reichlich vorhanden.

Abzug: Am 22. IX. am Steilufer der Warthe sich durch Zickern vernehmlich machend und zwar mehrere Stücke; am 26. X. 1 Stück auf dem St. Adalberts-Kirchhofe; am 11. XI. 2 Stücke gegen Mittag im Vorgarten des Marien-Gymnasiums. Überwinternde Stücke wurden in diesem Jahre nicht wahrgenommen.

1914: Am 1. IV. 2 Stücke in dem Garten der Wolfsmühle zickernd und sichtbar, 3-4 Stücke an der Wartheseite des Kernwerks singend.

Auf dem Abzuge: 14. X. zickernd im Vorgarten des MarienGymnasiums, am 25. X. 1 Stück am Eingange zum Schilling.

Nach Kayser bei Lissa ziemlich häufig. 


\section{Erithacus cyanecula M. W. - Weifssterniges Blaukehlchen.}

1912: Die ersten beiden Blaukehlchen hörte ich am 31. III. um $61 / 4$ p. in der Nähe des alten Rennplatzes. Auch in diesem Jahre waren Blaukehlchen an den zusagenden Stellen im Warthetale überall vertreten. Aufserdem fand ich einzelne Vögel: In dem Ausschachtungsgelände vor dem ehemaligen Eichwaldtore (am 21. IV. bruchstückweiser Gesang zu hören), im Buschwerk auf den Wiesen bei Johannistal, im Weidicht an der Rückseite der Schiefsstände vor dem Warschauer Tor (am 21. IV. um 6 Uhr p. lebhaft singend), in einer buschreichen Senkung jenseit der Loncz-Mühle. Dieses ơ liefs am 27. IV. immer wieder gewisse Meisenrufe (zídeh und zizidä) mit aller Deutlichkeit hören, und am 9. V. gab hier ein Blaukehlchen um $1 / 26$ p. den Gesang des Weidenlaubvogels sehr gut wieder; nur die Stimme war etwas kräftiger.

Am 7. VI. warnte gegen $6 \mathrm{Uhr}$ p. ein $\bigcirc$ ängstlich an einem der Tümpel der alten Warthe. Es hatte hier offenbar Junge.

Letzter Gesang am 12. VI. um $8 / 46$ p. an der Insel.

1913: Am 30. III. liefs ein schönes $\sigma^{x}$ um $1 / s 10$ a. in einigen Weidenbüschen vor dem ehemaligen Eichwaldtor trotz des scharfen Ostwindes bruchstückweisen Gesang hören; abends um 8/.7 sang 1 Stück im Weidengebüsch der Wiesen bei Johannistal.

Sonstiges Vorkommen: Am 17. IV. sah ich um $3^{20}$ am Schwersenzer See in einem einzelnen Weidenbusche 1 Stück, das sich offenbar noch auf dem Zuge befand; am 28. IV. traf ich mehrere Stücke in der Nähe des kleinen Ketscher Sees und an der Samica, die fleifsig sangen, also wohl Brutvögel sein mochten.

In der zweiten Hälfte des Mai und im Juni (bis zum 20.) sangen die Blaukeblchen in diesem Jahre besonders eifrig im Weidicht an der Warthe. Wahrscheinlich hatte dies darin seinen Grund, dafs die ersten Bruten durch die Überschwemmung Ausgangs April und Anfang Mai vernichtet worden waren, so dafs die Vögel zu einer Ersatzbrut hatten schreiten müssen.

Am 14. V. liefs $1 \sigma^{x}$ an der Warthe diesseit des Eichwaldes ein oftmaliges Zjep - - - - hören, das ähnlich klang wie der Paarungsruf der Sumpfmeise.

1914: Am 19. IV. 1 Stück in der Näbe der städtischen Flufsbadeanstalt im Weidengebüsch sichtbar; am 20. IV. 1 Stück am rechten Wartheufer gegenüber der Gärtnerei von Zippel in den vom Hochwasser freien Weidenbüschen singend; am 22. IV. $1 \sigma^{x}$ an einem Graben am Steilufer der Warthe, ein zweites in der Höhe der Wolfsmühle; das letztere liefs mehrmals das Sizidä der Kohlmeise hören. Im Juni noch mehrfach singend: Am 7. VI. $1 \sigma^{*}$, auch sichtbar, im alten Warthebett oberhalb der Wolfsmühle; am 10. VI. $2 \sigma^{x}$, etwa $100 \mathrm{~m}$ von einander entfernt, an dem rechten Wartheufer in der Höhe des Schillings gegen 8 Uhr p. 
singend, 2 weitere oberhalb der Cybinamündung; 1 Stück an der Einmündung des Vorflutgrabens; am 13. VI. um $1 / 88$ p. $10^{x}$ an der alten Warthe, auch sichtbar; am 16. VI. 1 of um 7 Uhr p. auf der Insel. Mehrere Flugzeuge zogen ratternd überbin, ohne dafs die singenden Blaukehlchen oder Schwalben und Segler dadurch irgendwio beunruhigt wurden.

Von Szulczewski wurden Blaukehlchen in Birkholz im Kreise Znin beobachtet (Schulz).

\section{Erithacus luscinia L. - Nachtigall.}

1912: Am 28. IV. sang um 4 Uhr p. 1 o $^{\top}$ an der Wartheseite des Viktoriaparks, ein zweites $\sigma^{T}$ an der Nordseite des Eichwaldes, beide treffliche Sänger; am 29. IV. um $4 \frac{1}{2}$ p. sang $1 \sigma^{\top}$ einige Strophen im Schilling, sonst war nirgends ein Vogel dieser Art zu hören (kühl bei 0. ; die Vögel mögen wohl schon am 27. oder am 26. eingetroffen sein, als noch Westwind wehte).

Reichlicher waren Nachtigallen erst am 7. V. vorhanden, so dafs ich an der Wartheseite des Kernwerks 10-11 Stücke zählen konnte. Ein Teil war sicherlich noch auf dem Zuge; denn ich traf um $5^{10} \mathrm{p}$. ein im Weidicht unterhalb der Wolfsmühle rastendes Stück, wo sonst diese Art nicht zu finden ist. Im ganzen genommen, waren die Nachtigallen in diesem Jahre bei Posen weniger zahlreich vertreten als sonst.

Am 15. VI. fand ich im Eichwalde ein Nest, das etwa $1 / 2 \mathrm{~m}$ hoch im Brombeergerank hing. Am 24. VI. brütete der Vogel noch, während sonst die Jungen schon fast flügge waren; am 1. VII. lagen Junge im Neste, die tüchtig sperrten. Die Brut ist offenbar aufgebracht worden, da das Nest bei meinem Besuche nach den Ferien sich als unbeschädigt erwies.

Am 27. VI. sang noch $10^{x}$ um 8 Uhr p. ziemlich eifrig und ein zweites um $8^{20} \mathrm{p}$. an der Wartheseite des Kernwerks.

Im August hört man vorzugsweise gegen Sonnenuntergang die Lockrufe der Nachtigallen. Nur einmal, am 10. VIII., rief 1 Stück sein Wihd um $4 \mathrm{Uhr}$ p. auf den Schiefsständen vor dem Warschauer Tor. Teilweise mögen es noch heimische Vögel aus Spätbruten sein; doch dürften auch wohl zugewanderte Stücke sich zusammenrufen, um sich zum Weitermarsche vorzubereiten. Soviel scheint sicher zu sein, dals die Vögel, wenn die Jungen völlig erwachsen sind, die Abreise antreten, da doch bei längerem Verweilen derselben mehr Vögel zu sehen und vor allem auch zu hören sein müfsten, als dies tatsächlich der Fall ist. Ich will auch diesmal die einzelnen Fälle, in denen ich im Laufe des Monats August Nachtigallen wahrnahm, hier anführen: Am 2. VIII. lockten um $1 /, 7$ p. an einer Stelle des Eichwaldes mehrere Stücke; am 6. VIII. war um $1 / 28$ p. an 4 Stellen der Wartheseite des Kernwerks das Wihd einzelner Vögel zu bören; am 9. VIII. riefen 2 Stücke ihr Wihd, mehrere liefsen ein leises, warnendes Krr 
hören; am 10. VIII. rief um 4 Uhr p. auf den Schiefsständen vor dem Warschauer Tor und um $1 / 87 \mathrm{p}$. in Kobylepole je ein Stück; am 12. VIII. um $7 \mathrm{Uhr}$ p. an der Nordwestseite des Kernwerks an mehreren Stellen lockend, ebenso um $1 / 68$. an der Wartheseite 1 Stück; am 14. VIII. gegen $1 / 88 \mathrm{p}$. an 3 Stellen der Wartheseite des Kernwerks je 1 Stück rufend; am 19. VIII. im Kernwerk nichts zu vernehmen; am 21. VIII. kurz nach 7 Uhr p. an 2 Stellen der Wartheseite des Kernwerks je 1 Stück wihd rufend und ein leises Krr anhängend; ebendort am 26. VIII. 1 Stück (oder 2) um $61 / 4$ p. Nach diesem Tage wurde keine Nachtigall mehr gesehen oder gehört.

1913: Am 25. IV. um $9^{20}$ a. im Goethepark ein singendes $\sigma^{\top}$, an der Südwest- und Südseite des Kernwerks etwa 5 Stücke, lauter gute Sänger; an der Ostseite des Kernwerks (Wartheseite) war noch kein Vogel zu hören (warm und schwül bei S., am 24. W. und SW.). Am 26. hatte sich die Zahl der singenden Vögel etwas vermehrt; auch auf den Kirchhöfen am Schillerpark 2 Stücke; am 30. daselbst 8-10 Stücke, im Goethepark mindestens 4 Stücke. Kayser hörte in Lissa, wo Nachtigallen häufig sind, gleichfalls am 25. IV. ersten Gesang.

Sonstiges Vorkommen: Am 3. V. in Schokken 2 singende or, in Lopuchowo ( $\mathrm{Kr}$. Obornik) 1 Stück; am 11. V. mehrere bei Obornik und einige im nahen Welnatale, darunter anscheinend ein Sprosser; am 13. V. mehrere bei Kurzagura (Kr. Kosten) in einer aus Kiefern, Fichten und Lärchen gemischten Schonung; am 17. V. im Tale der Gluwna und zwar bei Kowalskie 1 Stück, am Kowalskier See 3 Stücke, bei Jerzykowo 2 Stücke, bei OlschakMühle und etwas weiter oberhalb je 1 Stück.

Der Abzug ging in diesem Jahre vermutlich wegen der unfreundlichen Witterung im Juli recht früh vor sich. Am 3. VIII. wurde weder im Kernwerk noch zwischen Warschauer und Bromberger Tor von einer Nachtigall ein Ton vernommen, obwohl sie sich dort früher besonders gegen Sonnenuntergang durch ihr lockendes Wihd und das warnende Krr bemerkbar zu machen pflegten, so dafs sie kaum übersehen werden konnten. Auch in den folgenden Tagen war dort nichts von Nachtigallen zu spüren, auch nicht am Steilufer der Warthe. Nur 1 Stück liefs am 6. VIII. um $1 / 26 \mathrm{p}$. in einem Weidenbusche diesseit der Wartheinsel sein lockendes Whid hören und liefs sich ohne Scheu betrachten. Es war wohl ein Jungvogel. Sowohl beim Abzuge als beim Rückzuge wurden mehrfach Nachtigallen im Weidicht der Warthe beobachtet. Sie scheinen demnach auf ihrem Zuge teilweise der Warthe zu folgen, weil sie hier meist die erwünschte Deckung vorfinden. In der Brutzeit dagegen sind die Vögel dort nie zu treffen. Auch am 9. VIII. machte sich unterhalb des Schillings ein Durchzügler bemerkbar. Sonst konnte mit Sicherheit kein Vogel dieser Art mehr festgestellt werden. Die kühle,

Journ, f. Orn. LXVI. Jahrg. April 1918, 
regnerische Witterung hat offenbar die Vögel zur Beschleunigung ihres Abzuges veranlalst.

1914: Am 21. IV. kurz nach 7 Uhr p. warnte 1 Stück mehrmals mit wihd krr auf dem jenseit der Johanniskirche liegenden, an das Cybinatal anstofsenden Kirchhofe (Witterung sehr schön bei leichtem östlichen Winde); am 22. IV. liefs 1 Stück am Steilufer der Warthe einige Töne hören; am 23. IV. sang 1 Stück eine Strophe in den Anlagen vor dem Kalischer Tor; am 25. IV. 2 singende $\sigma^{x}$ im Schillerpark, 1 Stück an der Südseite des Kernwerks; am 26. IV. 1 singendes Stück unweit des Schillingtors; am 30. IV. sangen 8 Stücke an der Wartheseite des Kernwerks.

Auch Kayser beobachtete bei Lissa am 21. IV. das erste singende Stück.

Am 19. V. hörte ich in den neuen Solatscher Anlagen 5 bis $6 \sigma^{x}$ singen. Am 16. VI. fand ich ein etwa in Augenhöhe hinter Stammausschlägen einer Rüster stehendes Nest. Am 4. VIl. lagen Junge im Neste. Diese schienen aber nicht aufgekommen zu sein, denn nach den Ferien fand ich das Nest zerstört vor.

Am 1. VII. liefs noch $1 \sigma^{x}$ im Park von Solatsch einige Strophentrümmer hören, und auch am 4. VII. sang noch am Steilufer der Warthe 1 Stück mehrere Strophen.

Am 18. VI. hörte ich $1 \sigma^{x}$ im Warthewalde in der Nähe des Forsthauses singen, später 2 Stücke im Parke von Zerkow (Kr. Jarotschin).

1915: Erst am 30. IV. wurde gegen Abend Gesang dieser Art vernommen. Die Tage vorber waren kalt und unfreundlich.

\section{Erithacus philomela Bchst. - Sprosser.}

1912: Am 23. V. sang ein Sprosser an der Wartheseite des Kernwerks unweit des Schillingstors. Doch hat sich der Vogel hier nicht sefshaft gemacht. Am 31. V. hörte ich im Eichwalde um $71 /$ p. 1 Stück, jedoch nur dieses eine Mal an dieser Stelle. Dagegen traf ich am 13. VI. um 78/, p. einen Sprosser am Südrande des Eichwaldes, der sich als Standvogel erwies. Trotz des späten Termins seiner Entdeckung hörte ich den Vogel noch am 15. VI. um $1 / 26$ p., 19. VI. um $6^{10}$ p. und am 22 . VI. um $6^{3} / t$ und um $7 \frac{1}{4}$ p.; auch am 24. VI. sang der Vogel um $1 /, 7 \mathrm{p}$. noch ziemlich eifrig, während Nachtigallen nur noch hier und da bruchstückweisen Gesang hören liefsen. Seitdem aber habe ich ihn nicht wieder vernommen. Der Vogel war recht scheu und wich mir stets aus, so dafs ich ihn nicht zu Gesichte bekam. Doch war sowohl sein Gesang als auch sein Warnruf durchaus sprosserhaft.

1913: In diesem Jahre wurden 2 Sprosser im Eichwalde beobachtet und zwar an der Nord- und an der Südseite desselben. Am 14. V. liefs $1 \sigma^{x}$ an der nördlichen Randlache ausgesprochenen Sprossergesang hören. In der Folgezeit hatte sich der Vogel 
etwas tiefer in den Wald hineingezogen und etwa $30 \mathrm{~m}$ südlich der grofsen Lichtung seinen Standort gewählt. Hier sang der Sprosser am 27. V. um $1 / 27$ p. recht fleifsig, ebenso am 30 . V. um $4^{10} \mathrm{Uhr}$ p. Kaum 20 Schritt von ihm entfernt sang eine Nachtigall. Am 2. VI. um 6 und 7 Uhr p. hörte ich nur die letztere, während am 5. VI. um $4^{10} \mathrm{Uhr}$ p. bei einem heraufziehenden Gewitter sich beide in eifrigem Gesange zu überbieten suchten. Auch um $6^{\text {s0 }}$ sang der Sprosser eifrig. Seitdem habe ich ihn nicht wieder gehört.

An den vorber genannten Tagen habe ich auch das Stück an der Südseite des Eichwaldes verhört. Ich traf diesen Sprosser zuerst am 14. V. und zwar an derselben Stelle, an der auch im vorigen Jahre 1 Stück dieser Art gewohnt hatte. Ich hörte diesen Vogel am 18. VI. zum letzten Male. Auch bei den Nachtigallen hatte um diese Zeit die Sangeslust schon sehr merklich nachgelassen.

Sonstiges Vorkommen: Am 3. V. traf ich einen zwischen 4 und 5 Uhr p. eifrig singenden Sprosser unweit des Bahnhofs Revier (Kr. Wongrowitz) in der vom Wluknoer See sủdwärts ziehenden Senkung, die, eine richtige Sprossergegend, mit dichtem Weidicht bedeckt ist, in das halbhohe Birken eingestreut sind. Die westliche Seite dieser Senkung wird von einem stattlichen Kiefernhochwalde eingefafst, in dem Hohltauben sich durch Paarungsrufe bemerkbar machten. Am 11. V. traf ich im Welnatale bei Obornik einen Sänger, der anscheinend dieser Art angehörte.

1914: In diesem Jahre konnte mit Sicherheit kein Stäck dieser Art bei Posen nachgewiesen werden.

1915: Am 8. V. war am Nordrande des Eichwaldes Sprossergesang zu bören (tjojo $-\ldots-$ u. s. w.), ebendort auch am 17. V.

Einige Angaben über die Verbreitung des Sprossers in der Provinz habe ich im II. Bericht gemacht. Kayser hörte am 26. V. 1914 im Priebischer Bruch bei Lissa einen Sprosser.

$\mathrm{Zu}$ den vorher behandelten 221 Arten kommen noch folgende 7 in den früheren Berichten (I. oder II.) besprochene: Anas boschas L. - Stockente, Dendrocopus maior L. - Grofser Buntspecht, Dendrocopus minor L. - Kleinspecht, Passer domesticus L. - Haussperling, Passer montanus L. - Feldsperling, Acanthis cannabina L. - Bluthänfling, Parus caeruleus L. Blaumeise. Somit beträgt die Gesamtzahl der von mir bisher aus der Provinz Posen aufgeführten und besprochenen Arten und Nebenarten 228. 


\section{$2 \mathrm{BHL}$ Biodiversity Heritage Library}

1918. "Neuer Beitrag zur Kenntnis der Vogelwelt der Provinz Posen." Journal fu

r Ornithologie 66, 191-219. https://doi.org/10.1007/bf02286994.

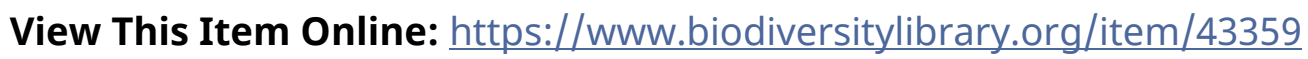

DOI: https://doi.org/10.1007/bf02286994

Permalink: https://www.biodiversitylibrary.org/partpdf/141921

\section{Holding Institution}

MBLWHOI Library

\section{Sponsored by}

MBLWHOI Library

\section{Copyright \& Reuse}

Copyright Status: No known copyright restrictions as determined by scanning institution.

This document was created from content at the Biodiversity Heritage Library, the world's largest open access digital library for biodiversity literature and archives. Visit BHL at https://www.biodiversitylibrary.org. 